$\begin{array}{ll}\text { Research Square } & \text { Preprints are preliminary reports that have not undergone peer review. } \\ \text { They should not be considered conclusive, used to inform clinical practice, } \\ \text { or referenced by the media as validated information. }\end{array}$

\title{
Group-Based Trajectory Modelling (GBTM) To Assess the Effect of Medication Adherence on Health-Related Outcomes: A Protocol for A Systematic Review
}

\section{Victoria Memoli ( $\nabla$ victoria.memoli@inserm.fr) \\ SESSTIM UMR 1252 https://orcid.org/0000-0001-6866-6675}

Giraud Ekanmian

Population Health and Optimal Health Practices Research Axis, CHU de Québec-Université Laval Research Center, CISSS de ChaudièreAppalaches Research Center

\section{Carlotta Lunghi}

Université du Quebec à rimouski; CISSS de Chaudière-Appalaches Research Center, Population Health and Optimal Health Practices Research Axis, CHU de Québec-Université Laval Research Center

\section{Anne-Déborah Bouhnik}

Aix Marseille Université, INSERM, IRD, SESSTIM, ISSPAM, Cancer, Biomedecine \& Society group, Equipe Labellisée Ligue Contre le Cancer, Marseille, France

\section{Sophie Lauzier}

Population Health and Optimal Health Practices Research Axis, CHU de Québec-Université Laval Research Center

Line Guénette

Population Health and Optimal Health Practices Research Axis, CHU de Québec-Université Laval Research Center

\section{Protocol}

Keywords: GBTM, Group-Based Trajectory Modelling, Group-Based Trajectory Modeling, Medication adherence, Latent class analysis, LCA, Latent Class Growth Analysis, LCGA, Health-related outcomes

Posted Date: June 8th, 2021

DOI: https://doi.org/10.21203/rs.3.rs-565670/v1

License: (c) (7) This work is licensed under a Creative Commons Attribution 4.0 International License. Read Full License 


\section{Abstract}

Background: The Group-based trajectory modelling (GBTM) method is increasingly used in pharmacoepidemiologic studies to describe medication adherence trajectories over time. However, assessing the effects of these medication adherence trajectories on health-related outcomes remains challenging. The purpose of this review is to describe studies assessing the effects of medication adherence trajectories estimated by the GBTM method on health-related outcomes.

Methods: We will conduct a systematic review according to the recommendations of the Preferred Reporting Items for Systematic Review and Meta-analysis (PRISMA) guidelines. We will search in the following databases: PubMed, Embase, PsycINFO, Web of Science, CINAHL, and Cochrane database up to April 1st, 2021. Two reviewers will independently select articles and extract data. Discrepancies at every step will be resolved through discussion, and consensus will be reached for all disagreed articles. A third reviewer will act as a referee if needed. We will use tables to synthesize the modalities used to estimate medication adherence trajectories and the effect of adherence trajectories on health-related outcomes. We will identify the types of health-related outcomes studied and how they are defined, the statistical models used, the effect measure yield, and how medication adherence trajectories have been incorporated in the model. We will also review the limitations and biases reported by the authors and their attempts to mitigate them. We will provide a narrative synthesis.

Discussion: This review will provide a clear view of the strategies and methods used in medication adherence research to estimate the effects of adherence trajectories on different health-related outcomes. A thorough exploration of how GBTM is used for this specific purpose could represent the first crucial steps towards optimizing the utilization of this method in adherence studies.

Systematic review registration: Prospero CRD42021213503.

\section{Background}

Medication adherence is a real challenge for healthcare professionals and patients. Sub-optimal adherence may be associated with poorer healthrelated outcomes and higher healthcare costs, depending on the disease.[1-3] Numerous adherence studies have been conducted to identify patients' characteristics with suboptimal adherence and the impact of non-adherence on health-related outcomes.[4] Several measures of adherence exist. When using medico-administrative databases as the source of drug information, researchers often use the proportion of days covered (PDC) or medication possession ratio (MPR), usually dichotomized with an $80 \%$ cut-off to distinguish adherent from non-adherent individuals.[4-6] Other adherence measures include self-reported questionnaires (e.g., Medication Adherence Report Scale (MARS)), tablet counts, electronic devices for vial caps) and more direct methods such as directly observing medication administration or dosage of drug metabolites in blood.[7-10] Most of these measures allow summarizing adherence over a definite period, ranging from few weeks to years. However, an individual's drug adherence may vary considerably over time, and a given summarized measure may recover situations drastically different from each other.

Studying adherence dynamics over time in a population may reflect patient's adherence behaviors more accurately than summarizing adherence as a single average measure over time. The Group-based trajectory modelling (GBTM) is increasingly used to describe the dynamic and mutable nature of medication adherence behaviors.[11-14] It is a statistical method for modelling a variable of interest throughout time or age by identifying groups of individuals with similar profiles.[15] The method was initially developed by Nagin et al. to characterize developmental trajectories of criminal activities[16] and became prevalent for studying developmental trajectories in criminology, social science, and psychology. [17] GBTM is also increasingly used in medicine and clinical research to study the development of different mental health disorders, such as depression, attention deficit hyperactivity disorder, post-traumatic stress, or addiction,[17-20] and to capture heterogeneity in treatment response in clinical trials.[21] The GBTM method is part of the latent class growth analysis (LCGA) family and is a specialized application of the finite mixture models.[22-24] [15]As described by Nagin et al., the basic model yields two results: first, it identifies individuals with similar trajectories (i.e., a group), and second, it estimates the individuals' probability of being part of each group.[25] For example, in medication adherence, the model allows to identify several adherence trajectories, each of them defining a specific adherence group (e.g., optimal adherence for the entire duration of the treatment, early discontinuation, suboptimal adherence throughout the treatment course, etc.). Each individual is then allocated to the trajectory that he/she has the highest probability to belong to. Once groups are identified, trajectories are plotted and presented in graphical form. Trajectories can also be used as the dependent variable to estimate characteristics associated with trajectory membership. Extensions of the classical GBTM model have been made, such as the possibility to account for risk and protective factors (e.g., age, sex, gender).[17] These analyses are relatively widespread as they are well described in the literature and benefit from software macros to help and guide researchers.[25, 26]

Medication adherence trajectories issued from GBTM could be beneficial for studying the associations between adherence and health-related outcomes, such as hospitalizations, death, or any critical clinical event. However, the GBTM literature does not provide specific instructions for assessing these associations. To our knowledge, there is no software macro developed to perform analyses combining trajectories and the outcome in a joint model. Researchers generally proceed in two steps; identifying the adherence trajectories with GBTM and then using these trajectories as an observed variable in any suitable model to infer health-related outcomes.

Page 2/16 
Nonetheless, this approach presents statistical challenges, such as the uncertainty associated with group membership. The groups identified with the GBTM method are probabilistic. The group assignment may be considered a $100 \%$ imputation, possibly resulting in a not-quantifiable uncertainty when inference about these groups is made through regression. Another concern is non-identifiability since GBTM imputes group membership on a not sufficiently general model, resulting in attenuated estimates of the relationship between trajectories and health-related outcomes. All these problems are not specific to the GBTM method but all latent class modeling.[27] These challenges have been described in particular by Bakk et al.[28] and Nylund-Gibson et al.[29], who have tried to find solutions to limit these biases.

The lack of guidance on the modelling of adherence trajectories to estimate their effect on health-related outcomes may result in heterogeneity of the methods used. Thus, it is critical to investigate the different approaches existing in the literature, the biases, and the difficulties encountered when applying adherence trajectory models to the study of distal outcomes. To our knowledge, there is no systematic review of studies that have used the GBTM method to measure the effects of medication adherence trajectories on health-related outcomes.

The purpose of this review is to identify and describe the studies assessing the effect of medication adherence trajectories, estimated by GBTM methods, on health-related outcomes. We will document the different types of study designs used, methods used to identify adherence trajectories, health-related outcomes studied, statistic modelling and parameters used, and limitations acknowledged by the studies' authors and how they were addressed.

\section{Methods/design}

This review will be conducted according to the recommendations of the Preferred Reporting Items for Systematic Review and Meta-analysis (PRISMA) guidelines.[30] For this protocol, we followed the recommendations of PRISMA-p.[31] The filled checklist is available in Appendix A.

\section{Eligibility criteria}

This systematic review will include all studies estimating medication adherence trajectories with the GBTM method [25] and evaluating the effects of these trajectories on specified health-related outcomes. We will not include studies on adherence to recommendations other than drug therapy (e.g., diet or exercise). Inclusion criteria are summarized in Table 1, available in Appendix B.

Table 1

Study selection criteria

\section{Inclusion}

Medication Any study about medication adherence, estimated with any kind of method whether direct (e.g., pill count or plasmatic adherence measurement) or indirect (e.g., questionnaire, adherence measure using medication database such as the proportion of days' covered medication possessing ratio, medication gap).

Group- $\quad$ The group-based trajectory modeling a statistical methodology for modeling the evolution of medication adherence over

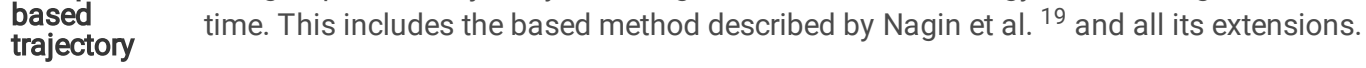

Adherence Adherence trajectory estimated with group-based trajectory as defined by Nagin et al. and considered as an independent trajectory variable in any statistic modeling.

Health Health-related outcomes any health results measured following an intervention or behavior (e.g., surgery, treatment etc.) that related describe a consequence of disease, treatment, or event for an individual. These health-related outcomes can be symptoms, outcomes hospitalizations, death, patient's quality of life, participation in activities, and social roles

Domain Health

Type of Original article,

document

Excluded: Conference abstract, commentaries, letter to editors, review. They will be nevertheless checked for references

Timeline No restriction

Language No restriction, document in other languages than English will be translated and extracted

Population No restriction

Inclusion Criteria

\section{Population:}

We will not apply restrictions to the population: all studies measuring medication adherence trajectories in any human population will be considered. We will not apply restrictions based on age, race, sex or gender.

\section{Intervention, exposure:}


We will consider any study in which medication adherence trajectories, defined by the GBTM method,[25] are used as an exposure variable to explore or estimate the effect of adherence on any specified health-related outcome.

\section{Comparators:}

Depending on the study design, no comparator may be required. We will not exclude any of the studies based on the comparator.

\section{Outcomes:}

We will allow for any health-related measure described in the study as an endpoint outcome (dependent variable) in relation to adherence trajectories.

\section{Study types:}

We will include all original studies, observational studies, randomized trials, quasi-experimental studies, cohort or case-control studies. Conference abstracts, commentaries, letters to editors, and reviews will be excluded but retrieved to identify potentially eligible references.

\section{Setting and time frame}

No limit will be set for the study setting or time frame. We will retain all the original studies, including those conducted in clinical settings or those part of an intervention or a trial. Selected articles will enter the initial screening stage without a time limit for execution or publication.

\section{Keys definitions}

\section{Medication adherence:}

Medication adherence is defined as "the extent to which patients follow the instructions given to them for prescribed treatments."[32] It is composed of three main concepts: 1) initiation (representing the extent to which a newly prescribed treatment is undertaken); 2) persistence (representing to what extent the treatment is taken for the recommended duration); and 3) implementation (representing to what extent the treatment is taken at the recommended doses and according to the recommended schedule).[33] We will consider all studies on medication adherence, whatever the type of adherence concept is measured.

\section{Adherence trajectory:}

Adherence trajectories are defined as descriptive longitudinal patterns of adherence over a defined time set. Defined adherence metrics measured over a short time set are allowed to vary over a longer predefined time horizon. Trajectories help to distinguish differences in patterns of adherence for individuals or groups of individuals over time.[11] Adherence trajectories model the evolution of adherence measures (for example, monthly PDC) over time and make it possible to identify people with similar adherence behaviours.[14]

\section{Health-related outcome}

\section{[34]}

Health-related outcomes are results measured following an intervention or behavior (e.g., surgery, treatment) and describe a consequence of disease, treatment, or event for an individual. These health-related outcomes can be symptoms, hospitalizations, death, patient's quality of life, participation in activities, and social roles. In medication adherence, these outcomes allow us to measure the effect and consequences of different adherence profiles on individuals.

\section{Information sources}

We will search for relevant article references in the following databases: PubMed, Embase, PsycINFO, Web of Science, CINAHL, and Cochrane database up to April 1st, 2021. The search strategy has been developed and adapted for each database using the most sensible approach validated by a specialized librarian at Laval University. A complete description of the applied search strategies is described in Table 2 in Appendix B. Duplicate citations will be removed using EndNote and Covidence Solution software.[35] A final manual revision of the database will be conducted to check for remaining duplicates. 
Table 2

Search strategy

\begin{tabular}{|c|c|c|c|}
\hline Strategy & Pubmed & Embase & Psyinfo \\
\hline $\begin{array}{l}\text { Treatment } \\
\text { adherence }\end{array}$ & $\begin{array}{l}\text { "treatment adherence and compliance"[MeSH Terms] OR } \\
\text { "adheren*"[Title/Abstract] OR "adheren*"[Other Term] OR } \\
\text { "complian**"[Title/Abstract] OR "complian*"[Other Term] OR } \\
\text { "persisten*"[Title/Abstract] OR "persisten*"[Other Term] OR } \\
\text { "nonadheren*"[Title/Abstract] OR "nonadheren*"[ Other Term] }\end{array}$ & $\begin{array}{l}\text { 'patient compliance'/exp } \\
\text { OR adheren*:ab,ti,kw OR } \\
\text { nonadheren*:ab,ti,kw } \\
\text { OR complian*:ab,ti,kw OR } \\
\text { noncomplian*:ab,ti,kw }\end{array}$ & $\begin{array}{l}\text { Treatment } \\
\text { Compliance/ OR } \\
\text { complian*.ti,ab,id OR } \\
\text { noncomplian*.ti,ab,idOR } \\
\text { persisten*.ti,ab,idOR }\end{array}$ \\
\hline \multicolumn{4}{|l|}{ Compliance } \\
\hline Adherence & $\begin{array}{l}\text { OR "noncomplian*"[Title/Abstract] OR "noncomplian*"[Other } \\
\text { Term] OR "nonpersisten*"[Title/Abstract] OR "nonpersisten** } \\
\text { [Other Term] }\end{array}$ & $\begin{array}{l}\text { OR persisten*:ab,ti,kw OR } \\
\text { nonpersisten*:ab,ti,kw } \\
\text { OR 'persistence':de }\end{array}$ & $\begin{array}{l}\text { nonpersisten*.ti,ab,id } \\
\text { OR adherenc*.ti,ab,id OR } \\
\text { nonadherenc*.ti,ab,id }\end{array}$ \\
\hline \multicolumn{4}{|l|}{ And } \\
\hline \multirow{2}{*}{$\begin{array}{l}\text { Group } \\
\text { based } \\
\text { trajectory } \\
\text { modelling }\end{array}$} & Trajectories[TIAB] & \multirow{3}{*}{$\begin{array}{l}\text { 'trajectory analysis':de OR } \\
\text { 'trajector* near/3 } \\
\text { model*':ab,ti,kw OR } \\
\text { 'gbtm':ab,ti,kw }\end{array}$} & \multirow{3}{*}{$\begin{array}{l}\text { Trajector*.ti,ab,id OR } \\
\text { GBTM .ti,ab,id }\end{array}$} \\
\hline & OR Trajectory[TIAB] & & \\
\hline $\begin{array}{l}\text { Trajectory } \\
\text { modelling }\end{array}$ & OR GBTM[TIAB] & & \\
\hline Strategy & Web of science & CINAHL & Cochrane database \\
\hline $\begin{array}{l}\text { Treatment } \\
\text { adherence }\end{array}$ & \multirow[t]{3}{*}{$\begin{array}{l}\text { ts }=(\text { adheren* or complian* or persisten* OR nonadheren* or } \\
\text { noncomplian* or nonpersisten*) }\end{array}$} & $\begin{array}{l}\text { MH medication compliance OR } \\
\text { AB (complian* or adheren* or } \\
\text { persisten*) OR AB ( } \\
\text { noncomplian* or nonadheren* }\end{array}$ & \multirow{3}{*}{$\begin{array}{l}\text { [mh "Treatment Adherence } \\
\text { and Compliance"] } \\
\text { OR(adheren*):ti,ab,kw OR } \\
\text { (nonadheren*):ti,ab,kw OR } \\
\text { (complian*):ti,ab,kw OR } \\
\text { (noncomplian*):ti,ab,kw } \\
\text { OR (persisten*):ti,ab,kw OR } \\
\text { (nonpersisten*):ti,ab,kw }\end{array}$} \\
\hline Compliance & & $\begin{array}{l}\text { or nonpersisten* }) \text { OR TI ( } \\
\text { complian* or adheren* or } \\
\text { persisten*) OR TI ( }\end{array}$ & \\
\hline Adherence & & $\begin{array}{l}\text { noncomplian* or nonadheren* } \\
\text { or nonpersisten*) }\end{array}$ & \\
\hline \multicolumn{4}{|l|}{ And } \\
\hline $\begin{array}{l}\text { Group } \\
\text { based } \\
\text { trajectory } \\
\text { modelling }\end{array}$ & \multirow[t]{2}{*}{ ts $=\left(\right.$ trajector ${ }^{\star}$ NEAR $/ 3$ model $\left.{ }^{\star}\right)$ OR ts $=(\mathrm{gbtm})$} & \multirow[t]{2}{*}{$\begin{array}{l}\text { TI (trajector* N3 model*) or AB } \\
\text { (trajector* N3 model*) OR TI } \\
\text { gbtm OR AB GBTM }\end{array}$} & \multirow[t]{2}{*}{$\begin{array}{l}\text { (trajector*):ti,ab,kw OR } \\
\text { (GBTM):ti,ab,kw }\end{array}$} \\
\hline $\begin{array}{l}\text { Trajectory } \\
\text { modelling }\end{array}$ & & & \\
\hline Search strat & & & \\
\hline
\end{tabular}

We will consider and include any additional articles not identified by our search strategy and brought to our attention by screening references of selected articles or relevant systematic reviews if they meet the inclusion criteria.

All identified studies will be compiled and kept with the full text (when needed) in a shared reference management software (i.e., EndNote)[36] and with Covidence, a web-based solution for systematic reviews.[35]

\section{Study selection}

Article selection will be performed using Covidence solution.[35] First, two reviewers will screen titles and abstracts independently, and articles will either be included, excluded, or categorized as unsure. Articles excluded by both reviewers will not be selected. Second, reviewers will discuss discrepancies to reach a consensus for every disagreed article. A pilot screening test will be conducted on a sample of a randomly selected $10 \%$ of the articles.

Likewise, the full text of selected articles will be reviewed independently by two reviewers, and articles will be included or excluded. The reason for exclusion will be documented. Discrepancies between the two reviewers will be resolved by discussion until an agreement is reached. A third reviewer will act as a referee if needed.

\section{Data extraction}

We will develop an extraction form using the Cochrane checklist of items to consider in data extraction.[37] The form will include the following elements:

- Study identification, including title, corresponding author's name and contact details, country, language, and publication date; 
- Study design and objectives;

- Health domain, including diseases and medications of interest;

- Sample size;

- Medication adherence measure used, including description of data source, adherence measure (e.g., adherence questionnaire, adherence measured from health database, medication electronic monitoring system), and a full definition of the variable and its operationalization (e.g., continuous, or dichotomized, threshold, scale, time frame);

- GBTM model software used, parameters, selection, and adequacy: link function, order, number of trajectories, statistics, and clinical criteria considered for the model selection.

- Health-related outcome definition: data source, variable definitions and their operationalization (e.g., continuous, dichotomized, time frame);

- Approach used in modelling the health-related outcomes (one step, two steps, or three steps) and the rationale;

- Model used to assess the effect of medication adherence trajectories on health-related outcomes and its description (e.g., linear regression, logistic regression, Cox modelling) and methods to take into account missing data, lost to follow-up, and censoring;

- Exposure variable: how trajectories were introduced in the model (e.g., group membership, inverse probability weighting);

- Limitations and biases identified by the authors;

- How authors tried to mitigate identified biases.

The form will be tested on a random sample of $10 \%$ of the included studies. We will contact study authors (three attempts, two weeks apart) to request any relevant missing information. Data extraction will be conducted independently by two reviewers, and discrepancies checked for accurate extraction.

The data form with item definitions is available in Table 3 in Appendix B. 
Table 3

Extraction Grid

\begin{tabular}{|c|c|c|}
\hline Variables & Definition & Format (or example) \\
\hline \multicolumn{3}{|l|}{ Identification } \\
\hline Id & Report the order number of the article assigned incrementally & 1 \\
\hline Author & $\begin{array}{l}\text { Report the name of the corresponding author as recorded in } \\
\text { the article }\end{array}$ & name, surname \\
\hline Mail & Report the corresponding author's mail & $x x x x @ x x x x . x x$ \\
\hline Year & Report the publication year of the article & yyyy \\
\hline Country & Report the country where the study has been conducted & \\
\hline Title & Report the title of the article & \\
\hline Study design & $\begin{array}{l}\text { Report the design of the study (Randomised trial, cohort } \\
\text { study, case-control, other) }\end{array}$ & \\
\hline \multicolumn{3}{|l|}{ Objectives } \\
\hline Objectives & $\begin{array}{l}\text { Report the objectives of the article as stated in the paper (copy } \\
\text { and paste) }\end{array}$ & \\
\hline \multicolumn{3}{|c|}{ Medication, intervention, analysis object } \\
\hline Intervention & $\begin{array}{l}\text { Report whether the study included an intervention } \\
\text { to improve adherence }\end{array}$ & $\begin{array}{l}\text { yes } \\
\text { no } \\
\text { unclear }\end{array}$ \\
\hline Medication class & Report the medication or class of medication of interest & $\begin{array}{l}\text { antidiabetic drugs } \\
\text { antihypertensive drugs } \\
\text { cardiovascular drugs } \\
\text { asthma drugs } \\
\text { oncology drugs } \\
\text { other, specify }\end{array}$ \\
\hline Medication other & $\begin{array}{l}\text { If other, specify the medication or class of medication of } \\
\text { interest }\end{array}$ & \\
\hline Medication's name & Report the name of all the medications mentioned & \\
\hline Prevalent or incident user & $\begin{array}{l}\text { Report whether the participants are new users or prevalent } \\
\text { users of the medication }\end{array}$ & $\begin{array}{l}\text { prevalent user } \\
\text { incident user } \\
\text { both } \\
\text { unclear }\end{array}$ \\
\hline Population disease & Report diseases of concern in the study & \\
\hline Population age group & $\begin{array}{l}\text { Specify whether there is any description of age group in the } \\
\text { study }\end{array}$ & \\
\hline Sample size & $\begin{array}{l}\text { Report whether the sample size calculation was } \\
\text { performed before the study }\end{array}$ & $\begin{array}{l}\text { yes } \\
\text { no } \\
\text { unclear }\end{array}$ \\
\hline Sample size calculation & $\begin{array}{l}\text { Report whether the sample size calculation was performed } \\
\text { considering the GBTM or the HRO }\end{array}$ & $\begin{array}{l}\text { power for GBTM } \\
\text { power for the HRO } \\
\text { both } \\
\text { other }\end{array}$ \\
\hline
\end{tabular}




\section{Variables}

Sample size method

\section{Adherence}

Adherence measure:

electronic monitoring caps (EMC) device

Adherence measure: adherence questionnaire

Adherence measure: adherence questionnaire

Adherence measure: adherence questionnaire type

Adherence measure: visual analog scale

Adherence measure: visual analog scale

Adherence measure: visual analog scale type

Adherence measured by

drug concentration/metabolite

levels (in the blood)

Adherence measured by pill count

Adherence measure: medico-administrative database

Adherence measure: claims database

Adherence measure: prescription database

Adherence measure in database: type

Report adherence measure used in the medicoadministrative database (EMC or similar device) adherence

Report the adherence questionnaire used

Report whether adherence is measured using a visual analog scale

Report whether the study has measured count administrative database database from prescription claims database from physician prescriptions
Report whether adherence is measured by an electronic device yes

Report whether an adherence questionnaire measures

Format (or example)

Report the method used to estimate the sample size

yes

no

Report the visual analog scale used by the author adherence using drug concentration/metabolite levels

Report whether the study has measured adherence by pill yes

no

Report whether the study computed adherence from a medico- yes

Report whether the study used a medico-administrative

no

yes

no

unclear

Report whether the study used a medico-administrative

yes

no

unclear

PDC

MPR

medication gap

other, specify

Adherence measure in database: other

If other, report the name of the adherence measure used in the database

Adherence measure in database: definition

Report whether the study reports a clear description of the adherence measure used

yes

no

unclear

Adherence measure in database: definition

Report the definition of adherence measure as stated in the document (copy and paste)

Adherence measure: time frame

Report whether the authors have indicated the period of measurement of adherence

yes

no

If yes, indicate the period of measurement of the adhesion (weekly, monthly, quarterly, etc.)

\section{GBTM}

GBTM: software

Report the software used for the GBTM

GBTM: software package

Report the package used 


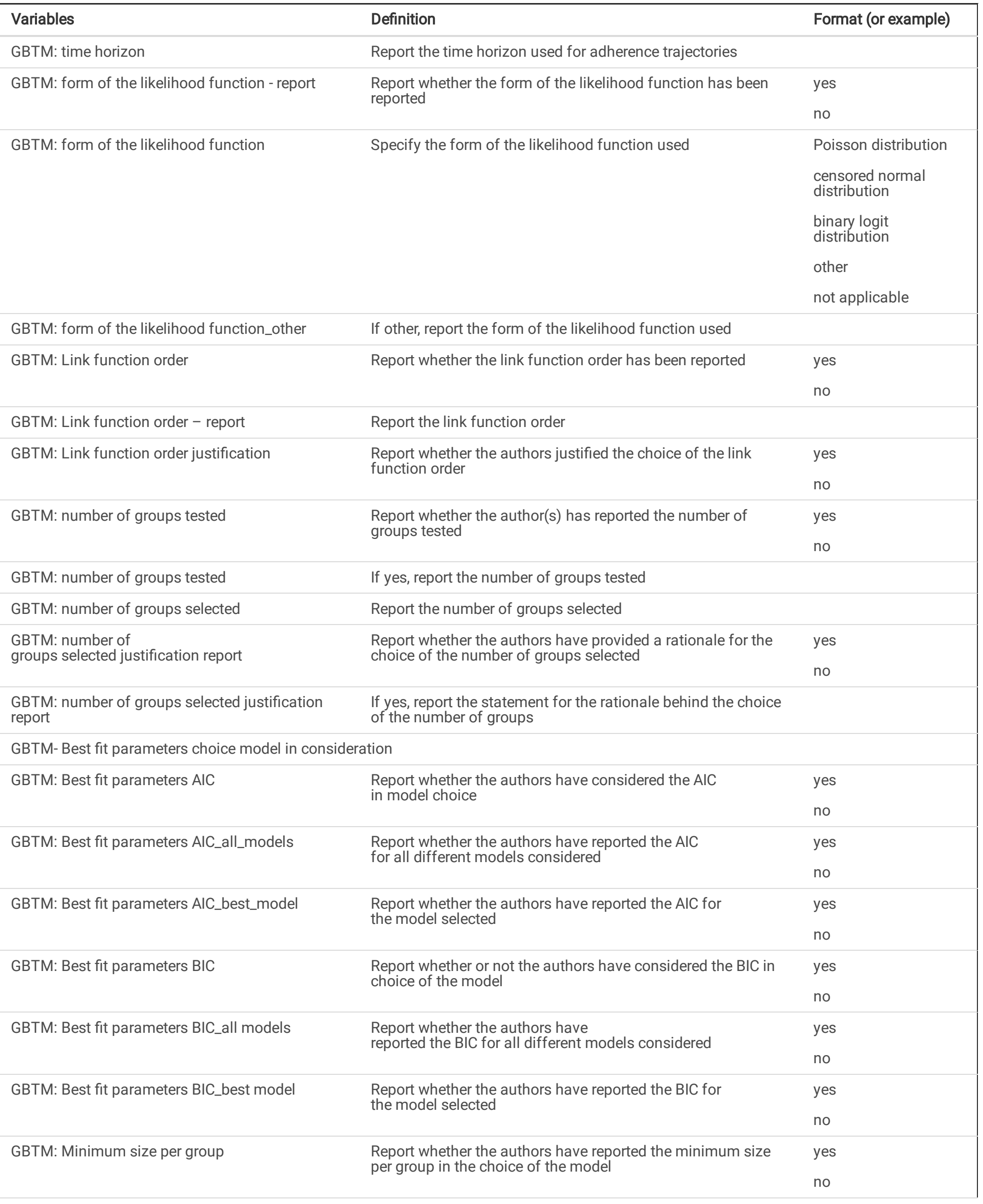




\begin{tabular}{|c|c|c|}
\hline Variables & Definition & Format (or example) \\
\hline $\begin{array}{l}\text { GBTM: Best-fit -Average Posterior Probability of } \\
\text { Assignment (APPA) }\end{array}$ & $\begin{array}{l}\text { Report whether the authors have considered the Average } \\
\text { Posterior Probability of Assignment (APPA) in choice of the } \\
\text { model }\end{array}$ & $\begin{array}{l}\text { yes } \\
\text { no }\end{array}$ \\
\hline GBTM: Best-fit -APPA all models & $\begin{array}{l}\text { Report whether the authors have reported the APPA for the } \\
\text { different models in consideration }\end{array}$ & $\begin{array}{l}\text { yes } \\
\text { no }\end{array}$ \\
\hline GBTM: Best-fit -APPA best model & $\begin{array}{l}\text { Report whether the authors have reported the APPA for the } \\
\text { model selected }\end{array}$ & $\begin{array}{l}\text { yes } \\
\text { no }\end{array}$ \\
\hline $\begin{array}{l}\text { GBTM: Best-fit -Odds of Correct } \\
\text { Classification (OCC) }\end{array}$ & $\begin{array}{l}\text { Report whether the authors have considered the Odds of } \\
\text { Correct Classification (OCC) in choice of the model }\end{array}$ & $\begin{array}{l}\text { yes } \\
\text { no }\end{array}$ \\
\hline GBTM: Best-fit -OCC- all models & $\begin{array}{l}\text { Report whether the authors have reported the OCC for the } \\
\text { different models in consideration }\end{array}$ & $\begin{array}{l}\text { yes } \\
\text { no }\end{array}$ \\
\hline $\begin{array}{l}\text { GBTM: Best-fit -Odds of Correct Classification-best } \\
\text { model }\end{array}$ & $\begin{array}{l}\text { Report whether the authors have reported the OCC for the } \\
\text { model selected }\end{array}$ & $\begin{array}{l}\text { yes } \\
\text { no }\end{array}$ \\
\hline $\begin{array}{l}\text { GBTM: Estimated Group Probabilities versus the } \\
\text { Proportion of the Sample Assigned to the Group }\end{array}$ & $\begin{array}{l}\text { Report whether the authors have considered the Group } \\
\text { Probabilities versus the Proportion of the Sample Assigned to } \\
\text { the Group in the choice of the model }\end{array}$ & $\begin{array}{l}\text { yes } \\
\text { no }\end{array}$ \\
\hline $\begin{array}{l}\text { GBTM- Best-fit- Estimated Group Probabilities } \\
\text { versus the Proportion of the Sample Assigned to } \\
\text { the Group-all models }\end{array}$ & $\begin{array}{l}\text { Report whether the authors have considered the Group } \\
\text { Probabilities versus the Proportion of the Sample Assigned to } \\
\text { the Group for the different models in consideration }\end{array}$ & $\begin{array}{l}\text { yes } \\
\text { no }\end{array}$ \\
\hline $\begin{array}{l}\text { GBTM- Best-fit- Estimated Group Probabilities } \\
\text { versus the Proportion of the Sample Assigned to } \\
\text { the Group-best model }\end{array}$ & $\begin{array}{l}\text { Report whether the authors have considered the Group } \\
\text { Probabilities versus the Proportion of the Sample Assigned to } \\
\text { the Group for the model selected }\end{array}$ & $\begin{array}{l}\text { yes } \\
\text { no }\end{array}$ \\
\hline $\begin{array}{l}\text { GBTM Best fit: Confidence Intervals for Group } \\
\text { Membership Probabilities }\end{array}$ & $\begin{array}{l}\text { Report whether the authors report the confidence intervals for } \\
\text { group membership probabilities }\end{array}$ & $\begin{array}{l}\text { yes } \\
\text { no }\end{array}$ \\
\hline GBTM Best fit: Tool for fit criteria assessment & $\begin{array}{l}\text { Report whether the authors have used a fit criteria } \\
\text { assessment tool for model selection }\end{array}$ & $\begin{array}{l}\text { yes } \\
\text { no } \\
\text { unclear }\end{array}$ \\
\hline GBTM Best fit: tool for fit criteria assessment & If yes, report the name of the tool & \\
\hline Analysis_Sensibility_GBTM & $\begin{array}{l}\text { Report whether the authors have conducted any sensibility } \\
\text { analysis for GBTM }\end{array}$ & $\begin{array}{l}\text { yes } \\
\text { no }\end{array}$ \\
\hline \multicolumn{3}{|l|}{ Missing Data } \\
\hline Missing Data & Report whether the authors considered missing data & $\begin{array}{l}\text { yes } \\
\text { no } \\
\text { unclear }\end{array}$ \\
\hline Missing data adherence & If yes, report how authors managed missing data in GBTM & $\begin{array}{l}\text { exclusion } \\
\text { imputation } \\
\text { other }\end{array}$ \\
\hline Missing data adherence_other & If other, specify & \\
\hline Missing data HRO & $\begin{array}{l}\text { If yes, report how authors managed missing data in HRO } \\
\text { modeling }\end{array}$ & $\begin{array}{l}\text { exclusion } \\
\text { imputation } \\
\text { other }\end{array}$ \\
\hline Missing data HRO_other & $\begin{array}{l}\text { If other, report how authors managed missing data in HRO } \\
\text { modeling }\end{array}$ & \\
\hline
\end{tabular}




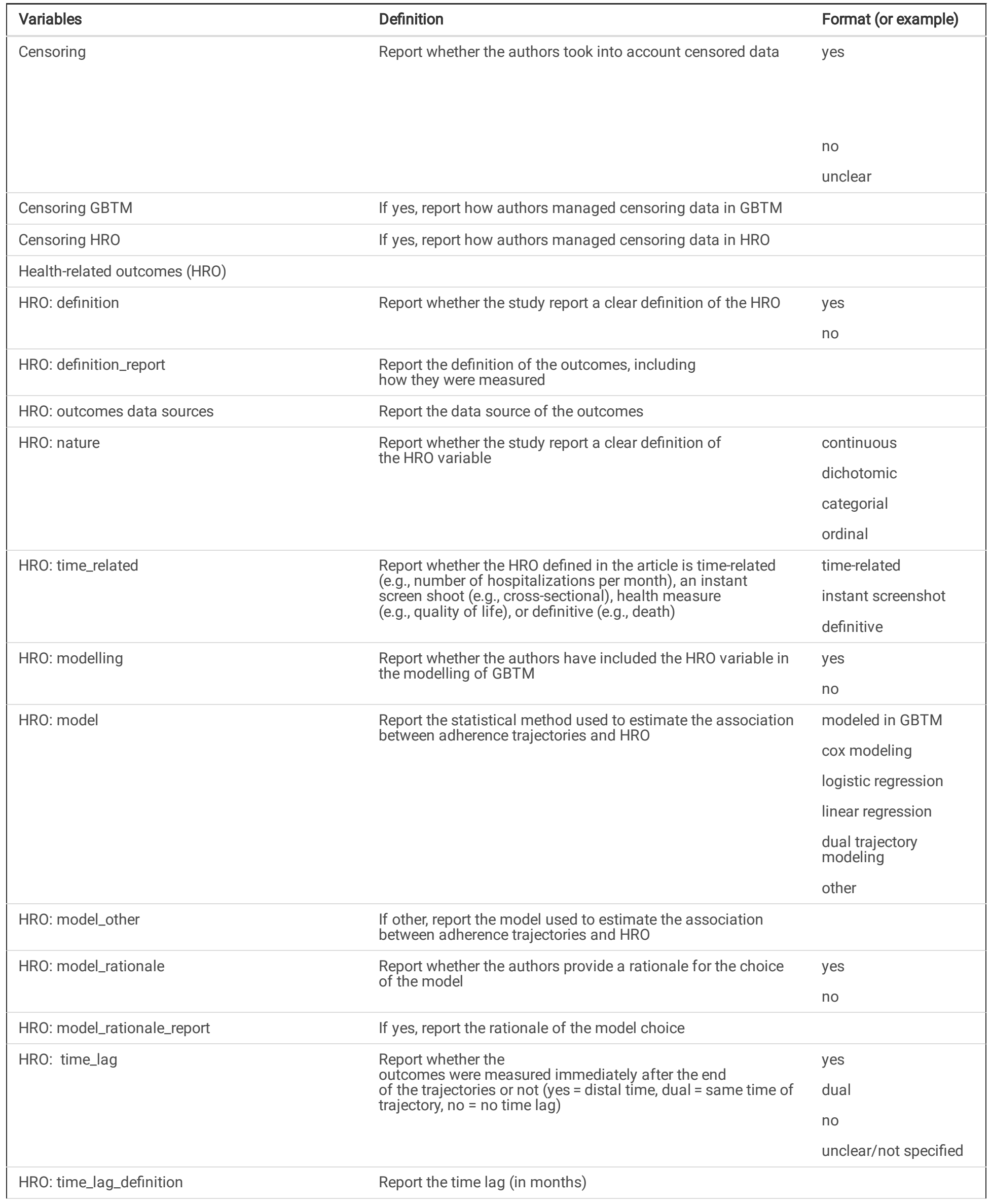




\begin{tabular}{|c|c|c|}
\hline Variables & Definition & Format (or example) \\
\hline HRO: time_lag_rationale & $\begin{array}{l}\text { Report whether the authors have provided a rationale for the } \\
\text { definition of the time lag used }\end{array}$ & $\begin{array}{l}\text { yes } \\
\text { no }\end{array}$ \\
\hline HRO: time_lag_rationale & If yes, report the rationale used & \\
\hline HRO: Adherence trajectories used & $\begin{array}{l}\text { Report how trajectories have been used in the } \\
\text { model (e.g., trajectory groups as a variable, inverse probability } \\
\text { weighting, e.tc) }\end{array}$ & \\
\hline Analysis_Sensibility_HRO & $\begin{array}{l}\text { Report whether the authors have conducted any sensibility } \\
\text { analysis for the HRO modelling }\end{array}$ & $\begin{array}{l}\text { yes } \\
\text { no }\end{array}$ \\
\hline \multicolumn{3}{|l|}{ Limitations } \\
\hline Confounding by indication & $\begin{array}{l}\text { Report whether the authors have reported confounding by } \\
\text { indication as a possible limitation of the study }\end{array}$ & $\begin{array}{l}\text { yes } \\
\text { no }\end{array}$ \\
\hline Time-dependent confounding & $\begin{array}{l}\text { Report whether the authors have reported time-dependent } \\
\text { confounding as a possible limitation of the study }\end{array}$ & $\begin{array}{l}\text { yes } \\
\text { no }\end{array}$ \\
\hline Healthy user/adherer effect & $\begin{array}{l}\text { Report whether the authors have reported healthy user/adherer } \\
\text { effect as a possible limitation of the study }\end{array}$ & $\begin{array}{l}\text { yes } \\
\text { no }\end{array}$ \\
\hline Protopathic bias & $\begin{array}{l}\text { Report whether the } \\
\text { authors have reported protopathic bias as a possible limitation } \\
\text { of the study }\end{array}$ & $\begin{array}{l}\text { yes } \\
\text { no }\end{array}$ \\
\hline Depletion of susceptible & $\begin{array}{l}\text { Report whether the authors have reported depletion of } \\
\text { susceptible as a possible limitation of the study }\end{array}$ & $\begin{array}{l}\text { yes } \\
\text { no }\end{array}$ \\
\hline Time-related bias & $\begin{array}{l}\text { Report whether the authors have reported time-related } \\
\text { bias as a possible limitation of the study }\end{array}$ & $\begin{array}{l}\text { yes } \\
\text { no }\end{array}$ \\
\hline Immortal time bias & $\begin{array}{l}\text { Report whether the authors have reported immortal time } \\
\text { bias as a possible limitation of the study }\end{array}$ & $\begin{array}{l}\text { yes } \\
\text { no }\end{array}$ \\
\hline Immeasurable time bias & $\begin{array}{l}\text { Report whether the authors have reported immeasurable time } \\
\text { bias as a possible limitation of the study }\end{array}$ & $\begin{array}{l}\text { yes } \\
\text { no }\end{array}$ \\
\hline Time-window bias & $\begin{array}{l}\text { Report whether the authors have reported time-window } \\
\text { bias as a possible limitation of the study }\end{array}$ & $\begin{array}{l}\text { yes } \\
\text { no }\end{array}$ \\
\hline Time-lag bias & $\begin{array}{l}\text { Report whether the authors have reported time- } \\
\text { lag bias as a possible limitation of the study }\end{array}$ & $\begin{array}{l}\text { yes } \\
\text { no }\end{array}$ \\
\hline Others & & \\
\hline Conflict of interest & Report whether the authors have reported conflict of interest & $\begin{array}{l}\text { yes } \\
\text { no }\end{array}$ \\
\hline
\end{tabular}

Quality assessment

The risk of bias and the quality of each study will be assessed using two checklists. For randomized trials, the risk of bias will be assessed with the Cochrane RoB 2.0 Tool.[38] For observational studies, we will use the ROBINS-I tool.[39] As the review does not intend to estimate a global measure of effect, no studies will be excluded based on the quality assessment. Quality assessment will only serve for analysis purposes and discussion of findings. This assessment will follow the same procedure as the data collection process. The quality assessment of each study will be done independently by two reviewers. Disagreements will be resolved by discussion between the reviewers or with a third reviewer as a referee.

\section{Assessment of reporting}

Included studies will be classified according to the quality of their reporting. The Detailed Guidelines for reporting on Latent Trajectory Studies (GRoLTS),[40] and the ESPACOMP Medication Adherence Reporting Guideline (EMERGE),[41] will be used to evaluate the studies. No study will be 
excluded during this step; instead, the reporting quality will be used for discussion purposes.

\section{Analysis}

Data analysis will proceed in three phases. In the first phase, we will describe selected studies with simple descriptive statistics and classify them in a table under the health domain studied (e.g., cancer, cardiovascular disease), medications used, population, and the studies' stated objective. In the second phase, we will summarize data according to the studies' method to estimate adherence trajectories. Studies will also be classified according to the source of data for medication adherence, medication adherence measure, the parameters used in GBTM, including the rationale behind parameter choice (e.g., statistics, clinical characteristics, the minimal number of patients included, number of groups). In the third phase, we will perform classification and narrative synthesis. We will review the choice and modalities used to estimate the effect of adherence trajectories on health-related outcomes, including the source of data, nature, and definition of the health-related outcomes studied, the statistical model used, the effect measure yield, and how medication adherence measure has been incorporated in the model. We will also summarize limitations and biases reported by the authors and their attempt to mitigate them. Moreover, we will classify studies according to the quality of reporting and the study's overall quality in the synthesis. As the review does not aim to estimate a measured effect, we will not conduct a metaanalysis and assess studies' heterogeneity.

\section{Discussion}

GBTM method has grown in popularity in adherence research over the last 20 years.[17] The method and its applications, the macroimplementations in the software, are well established and developed in many disciplines, such as pharmacoepidemiology.[42] Most of the studies on GBTM in this field have primarily used trajectories to describe membership groups over time and associated factors.[43, 44] To our knowledge, there are two systematic reviews on Latent Class Modelling approaches, including the GBTM method.[24, 45] Nevertheless, they did not focus on the GBTM method to assess the association between adherence trajectories and health-related outcomes and related challenges. While the GBTM method provides a more refined measure of medication adherence over time by identifying adherence trajectories,[46] it remains essential to study the effect of these trajectories on health-related outcomes. Despite the growing use of GBTM in adherence research and the availability of statistical tools, there is still considerable heterogeneity in how researchers use this method to model health-related outcomes.[47, 48] This again leads to disparate and sometimes confusing ways of studying and reporting outcomes. However, modelling health outcomes according to pathway groups could help identify problematic groups and subsequently guide interventions and policies. It is, therefore, necessary to review how the method is used to model data and how results are reported.

The review will summarize the various strategies and methods used by authors to estimate the effect of adherence trajectories on health-related outcomes. Special attention will be paid to study designs, model parameter specifications, and limitations. It will also document biases that could arise while using GBTM as an independent variable and how authors attempted to mitigate them. We will discuss how authors constructed the model, how they interpreted the results, and consider the effect of latent trajectories on health-related outcomes. Moreover, the review will also describe the studies' reporting quality with the two reporting guidelines specific to latent class analysis and adherence studies. Therefore, this review could represent the first crucial step towards developing a guide for the use of GBTM in medication adherence studies to infer healthrelated outcomes.

\section{List Of Abbreviations}

EMERGE ESPACOMP Medication Adherence Reporting Guideline

GBTM Group-Based Trajectory Modelling

GRoLTS The Detailed Guidelines for reporting on Latent Trajectory Studies

MPR Medication possession ratio

PDC Proportion of days covered

PRISMA Preferred Reporting Items for Systematic Review and Meta-analysis

Prisma-p Preferred Reporting Items for Systematic Review and Meta-Analysis Protocols

RoB 2.0 A revised tool to assess the risk of bias in randomized trials

ROBINS-I Risk Of Bias in Non-randomized Studies - of Interventions

\section{Declarations}


Ethics approval and consent to participate:

Not applicable

Consent for publication:

Not applicable

Availability of data and materials:

Not applicable

Competing interests:

The authors declare that they have no competing interests.

\section{Funding:}

GE have benefitted from a scholarship from the "Fonds d'enseignement et de recherche" of the Faculty of pharmacy of the Université Laval. He received the TD acceleration scholarship of the CISSS de Chaudière-Appalaches Research Centre jointly provided by the Fondation de I'Hôtel-Dieu de Lévis and TD bank. VM is supported by a doctoral grant from the Ligue nationale contre le cancer. SL is a research scholar with funding from the Fonds de recherche du Québec - Santé (Québec Health Research Fund).

\section{Authors' contributions:}

GE and VM conceived, designed the study protocol, and wrote the manuscript. SL, LG, ADB and CL contributed substantially to the conception and design of the study protocol and contributed significantly to the manuscript through a critical evaluation of the content and assisted with drafting the manuscript.

Acknowledgments: We would like to thank Sue-Ling Chang, who reviewed the manuscript for English.

\section{Authors' information:}

Victoria Memoli*. Aix Marseille Univ, INSERM, IRD, SESSTIM, ISSPAM, Cancer, Biomedicine \& Society group, Equipe Labellisée Ligue Contre le Cancer, Marseille, France victoria.memoli@inserm.fr

Giraud Ekanmian*. Population Health and Optimal Health Practices Research Axis, CHU de Québec-Université Laval Research Center, CISSS de Chaudière-Appalaches Research Center. codjo-giraud-ulrich.ekanmian.1@ulaval.ca

Carlotta Lunghi. Université du Québec à Rimouski; CISSS de Chaudière-Appalaches Research Center; Population Health and Optimal Health Practices Research Axis, CHU de Québec-Université Laval Research Center carlotta_lunghi@uqar.ca

Anne-Déborah Bouhnik. Aix Marseille Univ, INSERM, IRD, SESSTIM, ISSPAM, Cancer, Biomedicine \& Society group, Equipe Labellisée Ligue Contre le Cancer, Marseille, France anne-deborah.bouhnik@inserm.fr

Sophie Lauzier**. Population Health and Optimal Health Practices Research Axis, CHU de Québec-Université Laval Research Center Sophie.Lauzier@crchudequebec.ulaval.ca

Line Guénette**. Population Health and Optimal Health Practices Research Axis, CHU de Québec-Université Laval Research Center Line.Guenette@pha.ulaval.ca

\section{References}

1. Lauffenburger J, Acri T, Gross R. Studies of Medication Adherence. Pharmacoepidemiology [Internet]. John Wiley \& Sons, Ltd; 2019 [cited 2020 Nov 19]. p. 991-1009. Available from: http://onlinelibrary.wiley.com/doi/abs/10.1002/9781119413431.ch38

2. Verbrugghe M, Verhaeghe S, Lauwaert K, Beeckman D, Van Hecke A. Determinants and associated factors influencing medication adherence and persistence to oral anticancer drugs: a systematic review. Cancer Treat Rev. 2013;39:610-21.

3. Hutchins DS, Zeber JE, Roberts CS, Williams AF, Manias E, Peterson AM. Initial Medication Adherence-Review and Recommendations for Good Practices in Outcomes Research: An ISPOR Medication Adherence and Persistence Special Interest Group Report. Value Health. Elsevier; 2015;18:690-9.

4. Kardas P, Lewek P, Matyjaszczyk M. Determinants of patient adherence: a review of systematic reviews. Front Pharmacol. $2013 ; 4: 91$. 
5. Karve S, Cleves MA, Helm M, Hudson TJ, West DS, Martin BC. Good and poor adherence: optimal cut-point for adherence measures using administrative claims data. Curr Med Res Opin. 2009;25:2303-10.

6. Andrade SE, Kahler KH, Frech F, Chan KA. Methods for evaluation of medication adherence and persistence using automated databases. Pharmacoepidemiol Drug Saf. 2006;15:565-74; discussion 575-577.

7. Osterberg L, Blaschke T. Adherence to Medication. N Engl J Med. 2005;353:487-97.

8. Vrijens B, Geest SD, Hughes DA, Przemyslaw K, Demonceau J, Ruppar T, et al. A new taxonomy for describing and defining adherence to medications. Br J Clin Pharmacol. 2012;73:691-705.

9. Nguyen T-M-U, Caze AL, Cottrell N. What are validated self-report adherence scales really measuring?: a systematic review. Br J Clin Pharmacol. 2014;77:427-45.

10. McRae-Clark AL, Baker NL, Sonne SC, DeVane CL, Wagner A, Norton J. Concordance of direct and indirect measures of medication adherence in a treatment trial for cannabis dependence. J Subst Abuse Treat. 2015;57:70-4.

11. Franklin JM, Shrank WH, Pakes J, Sanfélix-Gimeno G, Matlin OS, Brennan TA, et al. Group-based Trajectory Models: A New Approach to Classifying and Predicting Long-Term Medication Adherence. Med Care. 2013;51:789-96.

12. Frank AS, Lupattelli A, Matteson DS, Nordeng H. Maternal use of thyroid hormone replacement therapy before, during, and after pregnancy: agreement between self-report and prescription records and group-based trajectory modeling of prescription patterns. Clin Epidemiol. 2018; Volume 10:1801-16.

13. Ajrouche A, Estellat C, De Rycke Y, Tubach F. Trajectories of Adherence to Low-Dose Aspirin Treatment Among the French Population. J Cardiovasc Pharmacol Ther. 2019;107424841986528.

14. Nicholas J, Edwards NC, Edwards RA, Dellarole A, Manca L, Harlow DE, et al. ND3 USE OF GROUP-BASED TRAJECTORY MODELING TO IDENTIFY ADHERENCE CLUSTERS IN PATIENTS WITH MULTIPLE SCLEROSIS NEWLY-INITIATING ONCE- OR TWICE-DAILY ORAL DISEASEMODIFYING DRUGS. Value Health. 2019;22:S43.

15. Nagin DS. Group-Based Trajectory Modeling: An Overview. Ann Nutr Metab. 2014;65:205-10.

16. Nagin DS, Land KC. Age, Criminal Careers, and Population Heterogeneity: Specification and Estimation of a Nonparametric, Mixed Poisson Model*. Criminology. 1993;31:327-62.

17. Nagin DS, Odgers CL. Group-based trajectory modeling in clinical research. Annu Rev Clin Psychol. 2010;6:109-38.

18. Jester JM, Nigg JT, Buu A, Puttler LI, Glass JM, Fitzgerald HE, et al. Trajectories of childhood aggression and inattention/hyperactivity: Differential effects on substance abuse in adolescence. J Am Acad Child Adolesc Psychiatry. 2008;47:1158-65.

19. Mora PA, Bennett IM, Elo IT, Mathew L, Coyne JC, Culhane JF. Distinct trajectories of perinatal depressive symptomatology: evidence from growth mixture modeling. Am J Epidemiol. 2009;169:24-32.

20. Orcutt HK, Erickson DJ, Wolfe J. The course of PTSD symptoms among Gulf War veterans: a growth mixture modeling approach. J Trauma Stress. 2004;17:195-202.

21. Brown $\mathrm{CH}$, Wang W, Kellam SG, Muthén BO, Petras H, Toyinbo P, et al. Methods for testing theory and evaluating impact in randomized field trials. Drug Alcohol Depend. 2008;95:S74-104.

22. McLachlan GJ, Lee SX, Rathnayake SI. Finite Mixture Models. Annu Rev Stat Its Appl. 2019;6:355-78.

23. JONES BL, NAGIN DS, ROEDER K. A SAS Procedure Based on Mixture Models for Estimating Developmental Trajectories. Sociol Methods Res. 2001;29:374-93.

24. Nguena Nguefack HL, Pagé MG, Katz J, Choinière M, Vanasse A, Dorais M, et al. Trajectory Modelling Techniques Useful to Epidemiological Research: A Comparative Narrative Review of Approaches. Clin Epidemiol. 2020;12:1205-22.

25. Nagin DS. Group-Based Modeling of Development. Cambridge, Mass: Harvard University Press; 2005.

26. Jennings W, Muniz C. Group-Based Trajectory Modeling. 2016.

27. Bray BC, Lanza ST, Tan X. Eliminating Bias in Classify-Analyze Approaches for Latent Class Analysis. Struct Equ Model Multidiscip J. 2015;22:1-11.

28. Bakk Z, Tekle FB, Vermunt JK. Estimating the Association between Latent Class Membership and External Variables Using Bias-adjusted Three-step Approaches. Sociol Methodol. SAGE Publications Inc; 2013;43:272-311.

29. Nylund-Gibson K, Grimm RP, Masyn KE. Prediction from Latent Classes: A Demonstration of Different Approaches to Include Distal Outcomes in Mixture Models. Struct Equ Model Multidiscip J. Routledge; 2019;26:967-85.

30. Moher D, Liberati A, Tetzlaff J, Altman DG, Group TP. Preferred Reporting Items for Systematic Reviews and Meta-Analyses: The PRISMA Statement. PLOS Med. 2009;6:e1000097.

31. PRISMA-P Group, Moher D, Shamseer L, Clarke M, Ghersi D, Liberati A, et al. Preferred reporting items for systematic review and meta-analysis protocols (PRISMA-P) 2015 statement. Syst Rev [Internet]. 2015 [cited 2020 Apr 30];4. Available from: https://systematicreviewsjournal.biomedcentral.com/articles/10.1186/2046-4053-4-1

Page 15/16 
32. Bissonnette JM. Adherence: a concept analysis. J Adv Nurs. 2008;63:634-43.

33. Grégoire J-P, Moisan J. Assessment of adherence to drug treatment in database research. In: Elseviers $M$, Wettermark B, Almarsdóttir AB, Andersen M, Benko R, Bennie M, et al., editors. Drug Util Res [Internet]. Chichester, UK: John Wiley \& Sons, Ltd; 2016 [cited 2019 July 15 th]. p. 369-80. Available from: http://doi.wiley.com/10.1002/9781118949740.ch36

34. Ward MM. CHAPTER 9B - Physical Function. In: Hochberg MC, Silman AJ, Smolen JS, Weinblatt ME, Weisman MH, editors. Rheum Arthritis [Internet]. Philadelphia: Mosby; 2009 [cited 2021 Feb 10]. p. 231-6. Available from:

https://www.sciencedirect.com/science/article/pii/B9780323054751500331

35. Covidence - Better systematic review management [Internet]. [cited 2020 Apr 30]. Available from: https://www.covidence.org/home

36. EndNote | Clarivate Analytics [Internet]. EndNote. [cited 2021 Jan 21]. Available from: https://endnote.com/

37. Higgins JPT, Green S. Cochrane Handbook for Systematic Reviews of Interventions. John Wiley \& Sons; 2011.

38. Risk of bias tools - RoB 2 tool [Internet]. [cited 2020 Apr 30]. Available from: https://sites.google.com/site/riskofbiastool/welcome/rob-2-0-tool

39. Sterne JA, Hernán MA, Reeves BC, Savović J, Berkman ND, Viswanathan M, et al. ROBINS-l: a tool for assessing risk of bias in nonrandomised studies of interventions. BMJ [Internet]. British Medical Journal Publishing Group; 2016 [cited 2020 April 30th];355. Available from: https://www.bmj.com/content/355/bmj.i4919

40. Schoot R van de, Sijbrandij M, Winter SD, Depaoli S, Vermunt JK. The GRoLTS-Checklist: Guidelines for Reporting on Latent Trajectory Studies. Struct Equ Model Multidiscip J. Routledge; 2017;24:451-67.

41. De Geest S, Zullig LL, Dunbar-Jacob J, Helmy R, Hughes DA, Wilson IB, et al. ESPACOMP Medication Adherence Reporting Guideline (EMERGE). Ann Intern Med. 2018;169:30-5.

42. Alhazami M, Pontinha VM, Patterson JA, Holdford DA. Medication Adherence Trajectories: A Systematic Literature Review. J Manag Care Spec Pharm. 2020;26:1138-52.

43. Modi AC, Rausch JR, Glauser TA. Patterns of nonadherence to antiepileptic drug therapy in children with newly diagnosed epilepsy. JAMA. 2011;305:1669-76.

44. Lambert-Côté L, Bouhnik A-D, Bendiane M-K, Bérenger C, Mondor M, Huiart L, et al. Adherence trajectories of adjuvant endocrine therapy in the five years after its initiation among women with non-metastatic breast cancer: a cohort study using administrative databases. Breast Cancer Res Treat. 2020;180:777-90.

45. van der Nest G, Lima Passos V, Candel MJJM, van Breukelen GJP. An overview of mixture modelling for latent evolutions in longitudinal data: Modelling approaches, fit statistics and software. Adv Life Course Res. 2020;43:100323.

46. Winn AN, Dusetzina SB. The association between trajectories of endocrine therapy adherence and mortality among women with breast cancer. Pharmacoepidemiol Drug Saf. 2016;25:953-9.

47. Jacobs P, Ang A, Hillhouse MP, Saxon AJ, Nielsen S, Wakim PG, et al. Treatment outcomes in opioid dependent patients with different buprenorphine/naloxone induction dosing patterns and trajectories. Am J Addict. 2015;24:667-75.

48. Mody A, Eshun-Wilson I, Sikombe K, Schwartz SR, Beres LK, Simbeza S, et al. Longitudinal engagement trajectories and risk of death among new ART starters in Zambia: A group-based multi-trajectory analysis. PLoS Med. 2019;16:e1002959.

\section{Supplementary Files}

This is a list of supplementary files associated with this preprint. Click to download.

- AppendixA.docx 